\title{
Lower Extracellular Glucose Level Prolonged in Elderly Patients With Severe Traumatic Brain Injury: A Microdialysis Study
}

\author{
Shoji YOKOBORI, ${ }^{1}$ Akihiro WATANABe, ${ }^{1}$ Gaku MATSUmoto, ${ }^{1}$ \\ Hidetaka ONDA, ${ }^{1}$ Tomohiko MASUnO, ${ }^{1}$ Akira FuSE, ${ }^{1}$ \\ Shigeki KUSHIMOTO, ${ }^{1}$ and Hiroyuki YOKOTA ${ }^{1}$
}

${ }^{1}$ Department of Emergency and Critical Care Medicine, Nippon Medical School, Tokyo

\begin{abstract}
Age may be an independent predictor of outcomes in traumatic brain injury (TBI), but the causes of the poor outcomes in elderly patients remain unclear. To clarify the differences between elderly and young patients with TBI, brain metabolism parameters were monitored with the microdialysis method in 30 patients with severe TBI (Glasgow Coma Scale scores 3-8). The microdialysis probe was inserted in the penumbra area of the brain and extracellular levels of glucose, glutamate, glycerol, lactate, and pyruvate were measured hourly for the initial 168 hours ( 7 days) after operation. The lactate/pyruvate ratio, which is considered to be a good indicator of neuronal ischemia, was also calculated. The patients were divided into the elderly group aged 65 years or older and the young group aged less than 65 years, and the biochemical markers were compared daily between these two groups. The value of extracellular glucose concentration was significantly lower in the elderly group than in the young group, and continued until the 7th day after injury. Moreover, the lactate/pyruvate ratio peaked on the 5th day after injury in the elderly group, later than in the young group. We concluded that neural vulnerability persisted longer in elderly patients than in young patients with TBI, and this should be considered to prevent the occurrence of additional secondary brain injury.
\end{abstract}

Key words: geriatric head injury, glucose, microdialysis, traumatic brain injury, hypoperfusion

\section{Introduction}

Traumatic brain injury (TBI) is very significant problem in the elderly population. TBI is responsible for more than 80,000 emergency department visits by persons aged 65 years or older each year in the United States, and this group has the highest rates of TBI-related hospitalization and death. ${ }^{29)}$ The Japan Neurotrauma Data Bank found significant differences in mortality rates between patients aged 65-69 years and patients aged 70-74 years. ${ }^{31)}$ Elderly individuals, 65 years of age or older, account for a disproportionate number of TBI cases and have a staggering incidence of morbidity and mortality. Older age is known to negatively influence outcome after TBI and the age may be the most important predictor of patient prognosis. ${ }^{10,14,18,21,22,26)}$ However, predicting outcomes and providing care in the older

Received November 9, 2010; Accepted January 25, 2011 adult population with TBI remains problematic without good clinical data.

Geriatric and neurotrauma investigators have identified many prognostic factors, but these variables remain understudied in older adults with TBI. Intracranial pressure (ICP), cerebral perfusion pressure (CPP), and jugular oxygen saturation $\left(\mathrm{SjO}_{2}\right)$ have been used in neurointensive care, and for decision making in neurotrauma treatment. In addition, cerebral microdialysis has been introduced as a neurochemical monitoring tool, ${ }^{27)}$ and is now routinely used in several neurointensive care units for bedside monitoring of small molecules related to brain perfusion and metabolism in neurotrauma patients. ${ }^{3)}$ However, any differences between elderly and young head injury patients in brain perfusion and metabolism measured with microdialysis remain unclear.

The present study investigated the differences between elderly and young TBI patients by monitoring of brain micro-metabolism with the microdialy- 
sis technique.

\section{Materials and Methods}

Ethical approval for the study was granted by the Ethical Committee of the Nippon Medical School Hospital. Patients were enrolled in the study in the emergency department and consented by proxy. The study involved 42 patients with severe TBI (Glasgow Coma Scale [GCS] scores 3-8) associated with acute subdural hematoma (ASDH) or diffuse brain injury (DBI) who were admitted to our emergency trauma center from January 1, 2009 to June 30, 2010. Consent from the families or proxy was not given for 9 patients. Three patients with epidural hematoma were excluded because of different clinical course and injury severity. Therefore, 30 patients (20 ASDH and $10 \mathrm{DBI}$ ) were finally enrolled in this study. No patient had additional injury including the trunk and extremities, and no history of serious preinjury use of anticoagulant and antiplatelet drugs, cardiovascular disease, diabetes mellitus, etc.

The initial management and operative procedures of the patients were performed with the protocols described in the Japanese Guidelines for the Treatment and Management of Severe Traumatic Brain Injury second edition, ${ }^{16)}$ and the Guidelines for the Management of Severe Traumatic Brain Injury third edition. ${ }^{5)}$ The patients remained in the neurointensive care unit for 7 days after surgery according to the above protocols.

After surgery for hematoma removal, a microdialysis probe (CMA70; CMA Microdialysis, Stockholm, Sweden), with a 10-mm membrane and 20,000-dalton molecular weight cut-off, and ICP monitor (Codman Microsensor; Codman \& Shurtleff, Inc., Raynham, Massachusetts, USA) were inserted into the white matter to $1-2 \mathrm{~cm}$ depth (so-called "brain penumbra area") from the brain surface with removed hematoma. ${ }^{3)}$ A CMA 103 perfusion pump (CMA Microdialysis) was connected in the operating theater, and normal saline was perfused through the catheter at a rate of $0.3 \mu \mathrm{l} / \mathrm{min}$, and the microdialysate was collected and frozen in 60minute samples for the initial 168 hours (7 days) after neurointensive care unit admission. The initial 60-minute sample was not used for analysis because this was the time allowed for stabilization of the probe.

Microdialysis was not interrupted for transport or bedside testing. The bedside nurse maintained a detailed patient event log to identify important events and to record times of vial sampling and all physiological monitoring data. The following data was recorded hourly: ICP, CPP, mean arterial pressure, heart rate, arterial oxygen saturation, core temperature (temperature in the rectal colon), and GCS score. In addition, doses of sedatives, mannitol, and other neurologically active medications were recorded hourly.

Frozen samples were briefly centrifuged and then analyzed on the ISCUS microdialysis analyzer (CMA Microdialysis) by batch analysis using standard ISCUS reagents. Extracellular levels of glucose, glutamate, glycerol, lactate, and pyruvate were measured, and the lactate/pyruvate ratio, which is considered to be a good indicator of neuronal ischemia, was also calculated in all samples.

The 30 patients were divided into the elderly group aged 65 years or older and the young group aged less than 65 years, and the biochemical markers were compared daily between these groups. Each case was treated with same target value of ICP and CPP. Statistical analysis was performed using Stat View software (SAS Institute Inc., Cary, North Carolina, USA). Differences in physiological parameters and biomarkers during the 7 days were analyzed by the Fisher exact test, Student's $t$-test, and one-factor analysis of variance. The analysis of significance between each day in every parameter used Scheffe's F test. The parameters are expressed as mean \pm standard deviation. The daily values of ICP, CPP, and chemical biomarkers were compared using the mean \pm standard error of the mean. The level of significance was taken as $\mathrm{p}<0.05$.

\section{Results}

The characteristics of the patient groups are shown in Table 1 . The mean age was $74.0 \pm 7.0$ years in the elderly group and $42.5 \pm 15.9$ years in the young group ( $p<0.0001$ ). The numbers of ASDH and DBI patients were the same in each group. Initial GCS score, blood pressure, body temperature, arterial blood gas examinations, and blood sugar showed no significant difference between the two groups. Good outcome (good recovery and moderate disability by the Glasgow Outcome Scale) was achieved in 4 of 15 patients in the elderly group and 9 of 15 patients in the young group, without significant difference $(\mathrm{p}=$ 0.0281).

The biochemical parameters are compared in the elderly and young groups in Table 2 . A total of 3,507 microdialysate samples $(1,560$ from the elderly group and 1,947 from the young group) were collected and analyzed. The overall mean glucose level was higher in the young group $(19.0 \pm 17.3 \mathrm{mmol} / \mathrm{l}$ in the young group, $15.3 \pm 20.0 \mathrm{mmol} / \mathrm{l}$ in the elderly group, $\mathrm{p}<0.0001$ ). The concentrations of glycerol, which indicate the degree of cell damage, were 
Table 1 Patient characteristics in the elderly and young groups

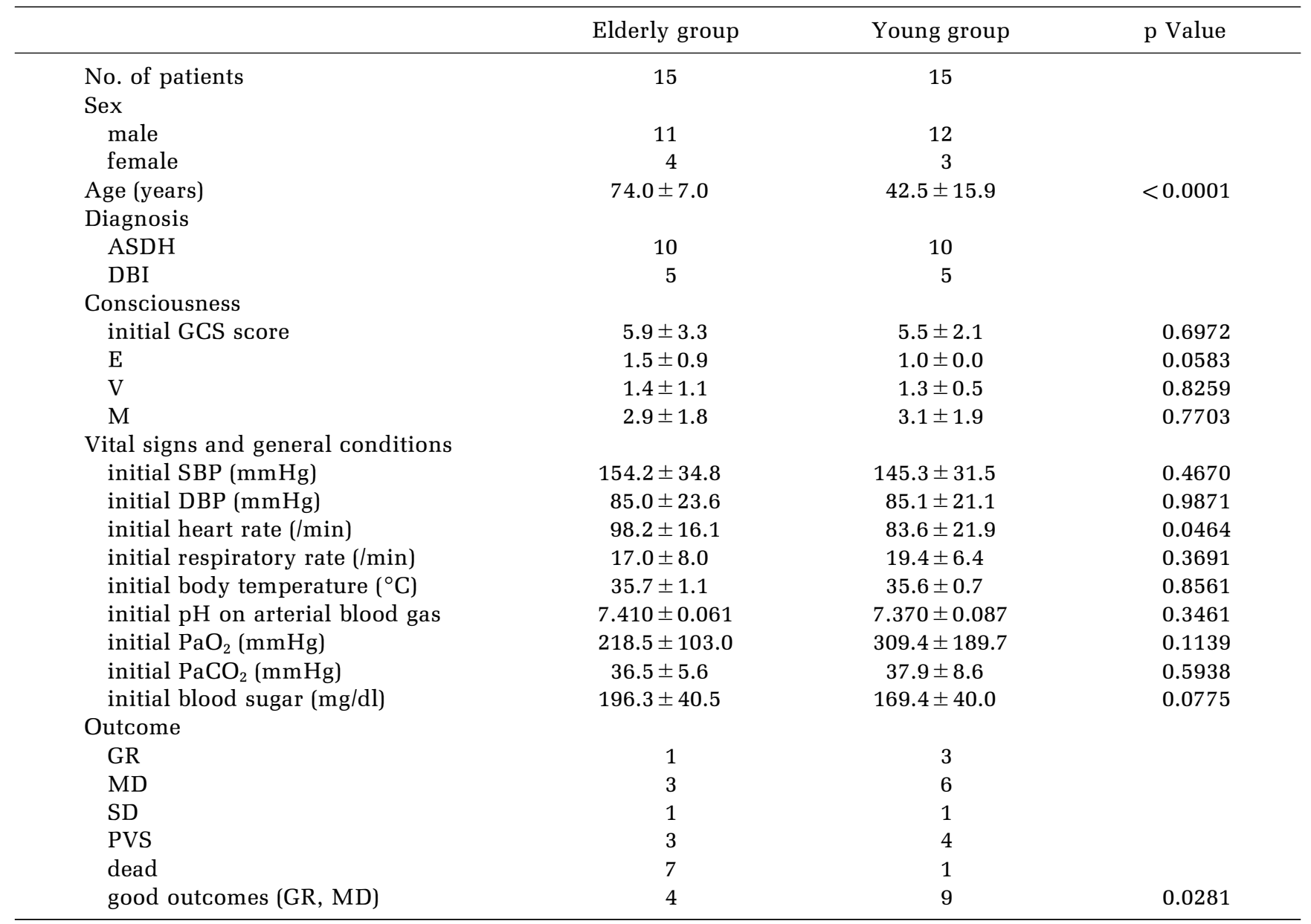

ASDH: acute subdural hematoma, DBI: diffuse brain injury, DBP: diastolic blood pressure, GCS: Glasgow Coma Scale, GR: good recovery, MD: moderate disability, PVS: persistent vegetative state, SBP: systolic blood pressure, SD: severe disability.

Table 2 Comparison of biochemical parameters between the elderly and young groups

\begin{tabular}{|c|c|c|c|c|}
\hline & $\begin{array}{l}\text { Normal value or } \\
\text { target value }\end{array}$ & Elderly group & Young group & $\mathrm{p}$ Value \\
\hline Number of samples & & 1560 & 1947 & \\
\hline Lactate $(\mathrm{mmol} / \mathrm{l})$ & $<2$ & $6.7 \pm 5.2$ & $7.1 \pm 6.5$ & 0.0472 \\
\hline Pyruvate (mmol/l) & $<0.12$ & $0.14 \pm 0.10$ & $0.15 \pm 0.11$ & $<0.0001$ \\
\hline Glycerol $(\mu \mathrm{mol} / \mathrm{l})$ & $<20-50$ & $681.6 \pm 755.8$ & $445.1 \pm 598.9$ & $<0.0001$ \\
\hline
\end{tabular}

LPR: lactate/pyruvate ratio.

higher in the elderly group. However, the lactate/pyruvate ratio showed no significant difference between the two groups. The extracellular concentration of glucose was lower and the lactate/pyruvate ratio was higher than the normal range ${ }^{25}$ in both groups.

Within the intensive care period of 7 days, the mean ICP remained in the range of $<20 \mathrm{mmHg}$ (Fig. $1 \mathrm{~A}$ ), and CPP remained in the range of $>70 \mathrm{mmHg}$ in both groups (Fig. 1B), showing good control wi- 

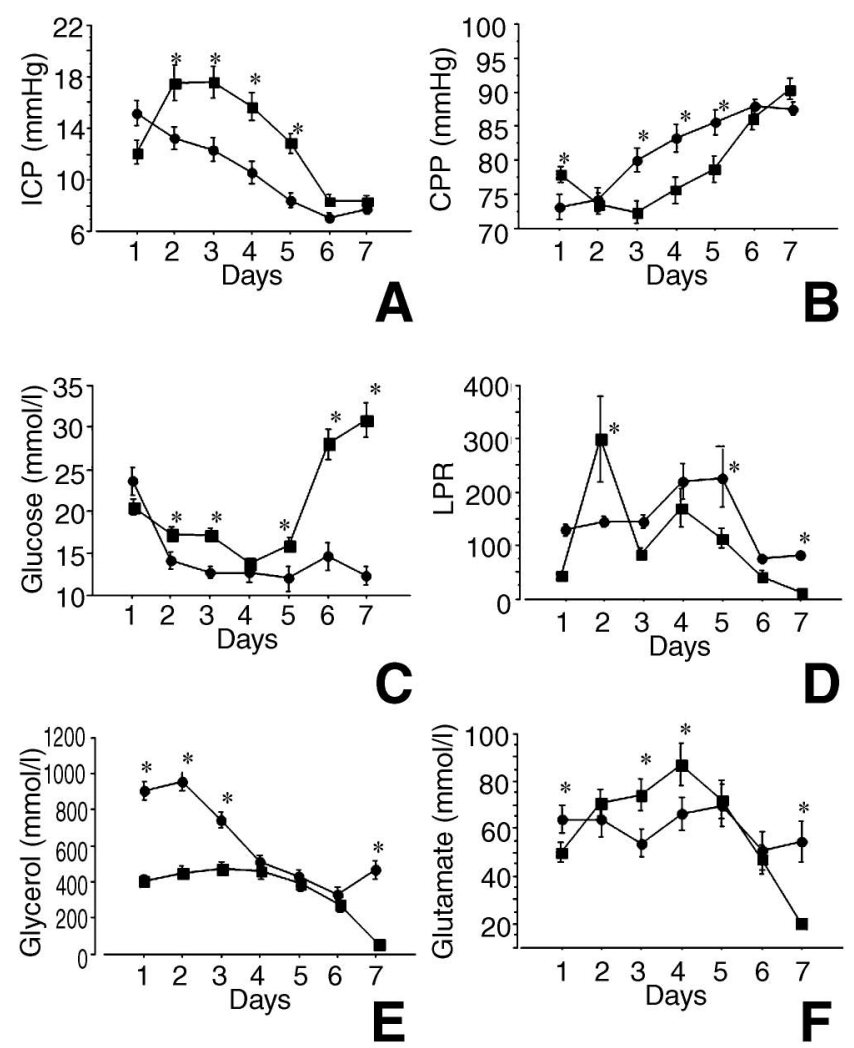

Fig. 1 Time courses of extracellular concentrations of biochemical parameters comparing the daily values in the young ( $<65$ years; squares) and elderly groups $(\geq 65$ years; circles) for 7 days after head injury. Daily values are expressed as mean \pm standard error of mean. ${ }^{*} p<$ 0.05, significant difference between elderly and young groups. CPP: cerebral perfusion pressure, ICP: intracranial pressure, LPR: lactate/pyruvate ratio.

thin the target limits of the treatment guidelines. The extracellular glucose concentration showed no significant difference between the elderly and young groups on the 1st hospital day, but was significantly lower in the elderly group than in the young group from the 2nd day until the 7 th day after injury (Fig. 1C). On the other hand, the extracellular glucose concentration recovered within 7 days in the young group (Fig. 1C). The lactate/pyruvate ratio gradually increased from the 1st day and peaked on the 5th day after injury in the elderly group, but peaked on the 2nd day in the young group (Fig. 1D). The extracellular concentrations of glycerol, which indicate neural cell damage, were significantly higher in the elderly group than in the young group at 3 days after injury, and continued for the remaining period (Fig. 1E). The extracellular glutamate concentrations peaked on the 4th day in the young group, but no abrupt change was observed in the elderly group (Fig. 1F). The mean glycerol and glutamate concen- trations gradually decreased in the young group, but significantly increased on the 7 th day in the elderly group (Fig. 1E, F).

\section{Discussion}

Our results demonstrated that lower extracellular glucose concentration persisted longer in elderly patients than in young patients after suffering TBI, and higher lactate/pyruvate ratio was prolonged in the elderly group. Moreover, the extracellular glucose concentration recovered to the normal range within 7 days in the young group, but remained lower than the normal range for 7 days in the elderly group.

Lower cerebral extracellular glucose concentration may be the consequence of several mechanisms, such as impaired glucose uptake, ${ }^{4)}$ or, in combination with high lactate/pyruvate ratio, may be a marker of increased anaerobic metabolism with hypoperfusion. ${ }^{13,15)}$ Lower extracellular glucose concentration was associated with higher lactate/pyruvate ratio in our elderly patients, so cerebral hypoperfusion may have persisted in elderly patients with head injury longer than in younger patients.

In our study, the mean CPP was maintained within the target range recommended in the guidelines. The CPP value was higher almost throughout the treatment course in elderly patients compared to young patients. However, cerebral hypoperfusion might have occurred in elderly patients with TBI. Cerebral extracellular glucose is a good indicator of the prognosis, ${ }^{24)}$ and levels of extracellular cerebral glucose are significantly less in patients with TBI who had poor outcome. ${ }^{33)}$ These findings together suggest that persistent cerebral hypoperfusion may underlay the persistent lower glucose concentration and poor outcome in elderly patients.

What are the causes of the cerebral hypoperfusion? One of the causes of insufficiency of cerebral perfusion may be that cerebral vascular pressure autoregulation is more impaired in elderly patients than in young patients. Cerebrovascular autoregulation is said to decrease with aging, and age-related decline in cerebrovascular autoregulation is associated with a relative deterioration in outcome in elderly TBI patients. ${ }^{6,7)}$ Therefore, we supposed that pressure autoregulation might be impaired longer in elderly patients than in young patients, and inadequate cerebral blood flow might lead to progression of secondary brain damage, as reflected by higher extracellular concentration of glycerol.

Serum thrombomodulin and von Willebrand factor levels, which are thought to be biochemical mar- 
kers reflecting injury of the cerebral vascular endothelium in TBI patients, were compared between elderly and young patient groups, and suggested that cerebral vascular endothelial injury would be more serious in elderly patients than in young patients. ${ }^{34)}$ As in our elderly patients, such vulnerability might be one of the reasons for long-term impairment of autoregulation and subsequent persistent cerebral hypoperfusion. The level of endothelial damage was higher in elderly patients than in younger patients, so the impairment of autoregulation might persist longer in elderly patients in our study.

To prevent secondary brain injury and improve outcome, accurate estimation of pressure autoregulation and adequate cerebral perfusion might be needed, especially in elderly patients. Continuous cerebrovascular autoregulation has been measured in the neurointensive care unit as correlation coefficients between arterial blood pressure and ICP, or the transcranial Doppler systolic flow velocity and ICP ${ }^{8,9)}$ The correlation between extracellular glutamate and CPP values was evaluated with the microdialysis method. Autoregulatory index, the correlation coefficient calculated by comparing glutamate concentration with CPP using Pearson's correlation, may be a good indicator for impaired or preserved autoregulation. ${ }^{27)}$ These techniques or equipments especially might be needed in the treatment of elderly TBI patients, and the efficacy of autoregulation-oriented intensive care should be analyzed in elderly patients with head injury.

Other factors may be related to decreasing extracellular glucose concentrations and increasing lactate/pyruvate ratio with mitochondrial damage in elderly patients. Deterioration of mitochondrial function has been proposed as a contributor to the aging process ${ }^{19,23,30,32)}$ and has been well characterized in pathological conditions. ${ }^{12)}$ Age-related mitochondrial dysfunction also occurs in traumatic brain-injured patients. ${ }^{1,2,11,28)}$ Age-related oxidative mitochondrial damage could accumulate in proteins responsible for importing substrates inside the mitochondria, affecting the enzymes need to start the tricarboxylic acid cycle, such as pyruvate dehydrogenase. ${ }^{17)}$ Such mitochondrial dysfunction might impair glucose transporting mechanisms and aerobic metabolism, and also extracellular glucose concentration and lactate/pyruvate ratio might be affected as in our study. Lower glucose concentration might occur as one of the features of persistent cerebral vulnerability in elderly TBI patients. Therefore, we should prevent progression of secondary brain injury in neurointensive care. Hypotension and hypoxia should be avoided with delicate intensive treatment, especially in elderly TBI patients.
In our study, there are some limitations about the technical factors. First, the biochemical measurement with microdialysis was restricted to only regional areas of the brain, and additional global cerebral monitoring $\left(\mathrm{SjO}_{2}\right.$, etc.) might be helpful for accurate cerebral perfusion and metabolism. Second, two types of injury (ASDH and DBI) were present in each group. The ratio of injury type was the same, but the mechanism might be different between ASDH and DBI. ${ }^{20)}$ More TBI cases should be analyzed with microdialysis in future investigations.

The main findings of this study are that the extracellular glucose concentration is lower in elderly patients than young patients with TBI and is associated with elevation of lactate/pyruvate ratio, which indicates progression of secondary brain injury, and with poor outcome in elderly TBI patients. Moreover, lower extracellular glucose persisted for 7 days after injury, and for longer in elderly patients than in young patients. Persistent low extracellular glucose level, associated with increasing lactate/pyruvate ratio and glutamate and glycerol levels, is considered to prolong secondary brain injury. We conclude that neural vulnerability persists for longer in elderly patients than in young patients with TBI, and we should consider these factors to prevent the occurrence of additional secondary injury.

\section{Acknowledgments}

We are in debt to the entire team that participated in data collection: Mrs. Mutsuko Hidenaga, Mrs. Reiko Takeuchi, and Mr. Yasushi Shibata in the laboratory department in Nippon Medical School Hospital, and all the nursing and research staff of the neurointensive care unit in Nippon Medical School Hospital.

This study was supported by a grant from the Foundation of General Insurance Association of Japan (2007).

\section{References}

1) Atamna H, Frey WH: Mechanisms of mitochondrial dysfunction and energy deficiency in Alzheimer's disease. Mitochondrion 7: 297-310, 2007

2) Beal MF: Mitochondria take center stage in aging and neurodegeneration. Ann Neurol 58: 495-505, 2005

3) Bellander BM, Cantais E, Enblad P, Hutchinson P, Nordström CH, Robertson C, Sahuquillo J, Smith M, Stocchetti N, Ungerstedt U, Unterberg A, Olsen NV: Consensus meeting on microdialysis in neurointensive care. Intensive Care Med 30: 2166-2169, 2004

4) Bergsneider M, Hovda DA, Shalmon E, Kelly DF, Vespa PM, Martin NA, Phelps ME, McArthur DL, 
Caron MJ, Kraus JF, Becker DP: Cerebral hyperglycolysis following severe traumatic brain injury in humans: A positron emission tomography study. J Neurosurg 86: 241-251, 1997

5) Brain Trauma Foundation; American Association of Neurological Surgeons; Congress of Neurological Surgeons; Joint Section on Neurotrauma and Critical Care, AANS/CNS, Bratton SL, Chestnut RM, Ghajar J, McConnell Hammond FF, Harris OA, Hartl R, Manley GT, Nemecek A, Newell DW, Rosenthal G, Schouten J, Shutter L, Timmons SD, Ullman JS, Videtta W, Wilberger JE, Wright DW: Guidelines for the management of severe traumatic brain injury. I. Blood pressure and oxygenation. J Neurotrauma 24 Suppl 1: S7-13, 2007

6) Chan TV, Ng SC, Lam JM, Poon WS, Gin T: Monitoring of autoregulation using intracerebral microdialysis patients with severe head injury. Acta Neurochir Suppl 95: 113-116, 2005

7) Czosnyka M, Balestreri M, Steiner L, Smielewski P, Hutchinson PJ, Matta B, Pickard JD: Age, intracranial pressure, autoregulation, and outcome after brain trauma. J Neurosurg 102: 450-454, 2005

8) Czosnyka M, Smielewski P, Kirkpatrick P, Laing RJ, Menon D, Pickard JD: Continuous assessment of the cerebral vasomotor reactivity in head injury. Neurosurgery 41: 11-19, 1997

9) Czosnyka M, Smielewski P, Kirkpatrick P, Menon DK, Pickard JD: Monitoring of cerebral autoregulation in head injured patients. Stroke 27: 1829-1834, 1996

10) Flaada JT, Leibson CL, Mandrekar JN, Diehl N, Perkins PK, Brown AW, Malec JF: Relative risk of mortality after traumatic brain injury: a population-based study of the role of age and injury severity. $J$ Neurotrauma 24: 435-445, 2007

11) Fukui H, Moraes CT: The mitochondrial impairment, oxidative stress and neurodegeneration connection: reality or just an attractive hypothesis? Trends Neurosci 31: 251-256, 2008

12) Gilmer LK, Ansari MA, Roberts KN, Scheff SW: Agerelated mitochondrial changes after traumatic brain injury. J Neurotrauma 27: 939-950, 2010

13) Hlatky R, Valadka AB, Goodman JC, Contant CF, Robertson CS: Patterns of energy substrates during ischemia measured in the brain in microdialysis. $J$ Neurotrauma 21: 894-906, 2004

14) Hukkelhoven CW, Steyerberg EW, Rampen AJ, Farace E, Habbema JD, Marshall LF, Murray GD, Maas AI: Patient age and outcome following severe traumatic brain injury: an analysis of 5600 patients. J Neurosurg 99: 666-673, 2003

15) Hutchinson PJ, O’Connell MT, Al-Rawi PG, Maskell LB, Kett-White R, Gupta AK, Richards HK, Hutchinson DB, Kirkpatrick PJ, Pickard JD: Clinical cerebral microdialysis: A methodological study. J Neurosurg 93: $37-43,2000$

16) Japan Society of Neurotraumatology, Committee of Guidelines for the Treatment and Management of Se- vere Traumatic Brain Injury: [Guidelines for the Treatment and Management of Severe Traumatic Brain Injury second edition]. Shinkei Gaisho Suppl 29: s15-84, 2006 (Japanese)

17) Long J, Wang X, Gao H, Liu Z, Liu C, Miao M, Liu J: Malonaldehyde acts as a mitochondrial toxin: inhibitory effects on respiratory function and enzyme activities in isolated rat liver mitochondria. Life Sci 79: 1466-1472, 2006

18) Luerssen TG, Klauber MR, Marshall LF: Outcome from head injury related to patient's age. A longitudinal prospective study of adult and pediatric head injury. J Neurosurg 68: 409-416, 1988

19) Merry BJ: Oxidative stress and mitochondrial function with aging - the effects of calorie restriction. Aging Cell 3: 7-12, 2004

20) Miller JD, Bullock R, Graham DI, Chen MH, Teasdale GM: Ischemic brain damage in a model of acute subdural hematoma. Neurosurgery 27: 433-439, 1990

21) Mosenthal AC, Lavery RF, Addis M, Kaul S, Ross S, Marburger R, Deitch EA, Livingston DH: Isolated traumatic brain injury: age is an independent predictor of mortality and early outcome. J Trauma 52: 907-911, 2002

22) Mushkudiani NA, Engel DC, Steyerberg EW, Butcher I, Lu J, Marmarou A, Slieker F, McHugh GS, Murray GD, Maas AI: Prognostic value of demographic characteristics in traumatic brain injury: results from the IMPACT study. J Neurotrauma 24: 259-269, 2007

23) Nicholls DG: Mitochondrial membrane potential and aging. Aging Cell 3: 35-40, 2004

24) Oddo M, Schmidt JM, Carrera E, Badjatia N, Connolly ES, Presciutti M, Ostapkovich ND, Levine JM, Le Roux P, Mayer SA: Impact of tight glycemic control on cerebral glucose metabolism after severe brain injury: A microdialysis study. Crit Care Med 36: 3233-3238, 2008

25) Reinstrup P, Ståhl N, Mellergård P, Uski T, Ungerstedt U, Nordström CH: Intracerebral microdialysis in clinical practice: Baseline values for chemical markers during wakefulness, anesthesia, and neurosurgery. Neurosurgery 47: 701-710, 2000

26) Signorini DF, Andrews PJ, Jones PA, Wardlaw JM, Miller JD: Predicting survival using simple clinical variables: a case study in traumatic brain injury. J Neurol Neurosurg Psychiatry 66: 20-25, 1999

27) Ståhl M, Bouw R, Jackson A, Pay V: Human microdialysis. Curr Pharm Biotechnol 3: 165-178, 2002

28) Swerdlow RH: Treating neurodegeneration by modifying mitochondria: potential solutions to a "complex" problem. Antioxid Redox Signal 9: 1591-1603, 2007

29) Thompson HJ, McCormick WC, Kagan SH: Traumatic brain injury in older adults: epidemiology, outcomes, and future implications. J Am Geriatr Soc 54: 1590-1595, 2006

30) Toescu EC, Verkhratsky A: Neuronal ageing from an intraneuronal perspective: roles of endoplasmic 
reticulum and mitochondria. Cell Calcium 34: 311-323, 2003

31) Tokutomi T, Miyagi T, Ogawa T, Ono J, Kawamata T, Sakamoto T, Shigemori M, Nakamura N: Age-associated increases in poor outcomes after traumatic brain injury: a report from the Japan Neurotrauma Data Bank. J Neurotrauma 25: 1407-1414, 2008

32) Trifunovic A, Larsson NG: Mitochondrial dysfunction as a cause of aging. J Intern Med 263: 167-178, 2008

33) Vespa PM, McArthur D, O’Phelan K, Glenn T, Etchepare M, Kelly D, Bergsneider M, Martin NA, Hovda DA: Persistently low extracellular glucose correlates with poor outcome 6 months after human traumatic brain injury despite a lack of increased lactate: A microdialysis study. J Cereb Blood Flow Metab 23:
865-877, 2003

34) Yokota H, Atsumi T, Araki T, Fuse A, Sato H, Kushimoto S, Koido Y, Kawai M, Yamamoto Y: Cerebral endothelial injury in elderly patients with severe head injury measured by serum thrombomodulin and von Willebrand factor. Neurol Med Chir (Tokyo) 47: 383-388, 2007

Address reprint requests to: Shoji Yokobori, MD, PhD, Department of Emergency and Critical Care Medicine, Nippon Medical School, 1-1-5 Sendagi, Bunkyo-ku, Tokyo 113-8603, Japan.

e-mail:shoji@nms.ac.jp 